\title{
A continuum model for entangled fibres
}

\author{
M. E. M. $\operatorname{LEE}^{1}$ and H. OCKENDON ${ }^{2}$ \\ ${ }^{1}$ School of Mathematics, University of Southampton, Highfield, Southampton SO17 1BJ, UK \\ email: lee@soton.ac.uk \\ ${ }^{2}$ OCIAM, Mathematical Institute, University of Oxford, 24-29 St. Giles', Oxford OX1 3LB, UK
}

(Received 23 December 2004; revised 9 February 2005)

\begin{abstract}
Motivated by the study of fibre dynamics in the carding machine, a continuum model for the motion of a medium composed of fibres is derived under the assumption that the dominant forces are due to fibre-fibre interactions and that the material is in tension. To characterise the material we include the averaged values of density and velocity and introduce variables to describe the mean direction, alignment and entanglement. We assume that the bulk stress of the material depends on the density, entanglement, degree of alignment, average direction and shear-rates. A kinematic equation for average direction and two proposed heuristic laws for the evolution of entanglement and degree of alignment are given to close the system. Extensional and shearing simulations are in good qualitative agreement with experimental results.
\end{abstract}

\section{Introduction}

\subsection{Industrial background}

The fundamental operations of yarn manufacture are carding, drawing, twisting and spinning. Carding transforms the raw material, such as cotton, wool or polyester, into a smooth coherent web disentangling, ordering and eliminating unwanted materials. The name for this process comes from the Latin for a thistle plant, as these were once used to tease and order handfuls of fibres. Although manufacturing technology has improved the speed and efficiency of the process, the underlying principles have not changed; now machines use huge arrays of hooks that travel at speeds of up to $100 \mathrm{~m} / \mathrm{s}$.

The forces applied to transform the disordered, entangled body of fibres during the carding process result in interconnected fibre structures being continually teased apart into individual fibres $[8,17]$. Therefore most of the activity results in permanent nonrecoverable changes in the internal orientation of the fibre medium. The transport of fibres relative to one another is crucial if one is to engage in good carding practice.

There are two detrimental effects of the carding process that undermine yarn manufacture. The first is that stresses generated in the machines can cause fibres to break: shorter fibres reduce the implicit strength of the resulting yarn. The second problem is the production of localised, highly tangled regions called neps, which result in dramatic density irregularities that are subsequently difficult to eliminate. Another inherent problem when dealing with textile fibres is the diversity of raw materials used in the industry. For example, the surfaces of fibres vary from smooth to crenulated, some fibres have frictional 
properties that depend on direction, and there are other differences in geometry and mechanics. With all this in mind the aim of the carding machine designers is to produce a single machine that can cope with any textile fibre and it is the understanding of this process and its dependence on the parameters that we seek.

\subsection{Modelling objectives}

In this paper we present a continuum model for an array of fibres that includes the evolution of fibre orientations and the degree of entanglement. Understanding how the fibre orientations change relative to one another during the carding process will aid the development of future machines and the optimisation of the process. This along with a number of other models of the carding process has been addressed by Lee [18].

\section{The model}

\subsection{Preamble}

Particles suspended in fluid have attracted the interest of applied mathematicians, especially the dilute or semi-dilute limits where particles do not interact or only interact hydrodynamically; see, for example, Batchelor [3] and Hinch \& Leal [13, 14]. Using a similar approach where the bulk stress consists of both hydrodynamic and particulate contributions Spencer [25] has modelled fibre-reinforced fluids. Toll et al. [26, 27, 28] considered densely packed fibres where the dominant forces are due to fibre-fibre interactions, although a planar constraint used in the derivation of the model is not applicable to fibre dynamics in the carding machine.

Other specific studies that include nematic particles such as polymers and liquid crystals are described by Doi \& Edwards [9] and Ericksen [10] and Leslie [19], respectively. The study of dense polymer chains and concepts such as the paradigm tube model consider large nematic molecules in a state of thermal agitation and so the underlying mechanics is quite different although the geometry is very similar to the problem considered here. Liquid crystal models employ two variables that we will later adopt: the mean crystal (in our context, fibre) direction, called the director, and the degree of crystal alignment, called the order parameter; our order parameter measures the degree to which the material has been carded. Although the director and order parameter are a very useful way of describing the material we cannot take the analogy with liquid crystals too much farther. In particular the internal forces here are quite different to those in liquid crystals and our approach to modelling the dynamics of the fibres is based on ideas from the theory of fibre suspensions.

Buckley [4] has suggested using an anisotropic visco-elastic continuum to represent the bulk stress of a fibre-medium. This means that the material has historical recovery and relaxation time-scales, the ratio of which forms the Deborah number. A number of authors [16, 22, 24] have employed the theory of dilute fibre suspensions, which can be applied to the parts of the carding process where entanglements have been eliminated and the density has been significantly reduced.

Another approach to modelling the carding process is to ignore "micro-scale" mechanics between individual fibres, and consider more global or "macroscopic" variables such as 
transfer rates between surfaces. A number of authors [1, 12, 23, 29] use a method that indicates the dynamics of fibre densities on each drum induced by prescribed transfer rates. Cherkassky $[5,6]$ employs a slightly more complicated model assuming a constant rate of transverse diffusion. For these models it is not easy to relate the theoretical parameters to specific physical parameters such as the fibres' frictional properties. Furthermore, being informed about transfer rates of fibres and even transverse diffusion does not immediately elucidate the dynamics of this textile process or the degree to which the raw material has been carded.

In this paper we derive a model that focuses on the disentangling and ordering of a many-fibre medium. The only significant internal forces are those between neighbouring fibres when they slip over one another as they re-orientate. Although the dynamic friction properties of textiles inevitably involve some ad-hoc constitutive laws, we will introduce a general framework for a dense, entangled, textile-fibre continuum. Besides using a director and an order parameter we will introduce a variable that characterises the entanglement of the medium.

\section{Governing equations}

We have chosen a continuum approach to derive a model which is tractable and applicable in the parts of the carding machine in which tufts of fibres are subjected to stress. As the industrial process involves large shear stresses, fibres will slip over one another, and we assume the material to be an anisotropic viscous continuum. We describe the fibre material with the following dependent variables and in all cases these are values averaged over a small volume containing a large number of fibres: density $\rho$, velocity $u_{i}$, average fibre direction or director $a_{i}$, order parameter $\phi$ and entanglement $\kappa$. The governing equations will be composed of a set of nine coupled Eulerian field equations that are: conservation laws for mass and momentum, a kinematic equation, and two constitutive evolution equations. However, before we go into the details of these equations we shall discuss two of the less familiar variables: order parameter $\phi$ and entanglement $\kappa$.

\subsection{Order parameter}

We adopt the order parameter from the theory of liquid crystals, as described by de Gennes $\&$ Prost [7]. If $\Theta$ is the angle between a typical fibre and the unit vector $a_{i}$, then the degree of alignment or the order parameter can be defined as

$$
\phi(\boldsymbol{x}, t)=\int_{S}\left(\frac{3 \cos ^{2} \Theta-1}{2}\right) \psi(\boldsymbol{x}, t, \Theta) d S,
$$

where $\psi$ is a probability density function that gives the probability that a fibre at position $\boldsymbol{x}$ makes an angle $\Theta$ with the director and $S$ is a unit sphere. This definition includes three distinct fibre orientations: $\phi=1$ requires all fibres to be aligned, $\phi=0$ means that fibre orientations are random and isotropic and $\phi=-\frac{1}{2}$ is planar isotropy. In our applications we expect that the order parameter will be non-negative since the fibres will be initially disordered $(\phi=0)$ and $\phi$ will increase as the material is sheared or stretched. Note that in the case where the fibres are isotropic, $\phi=0$, the director $\boldsymbol{a}$ is not well defined. 


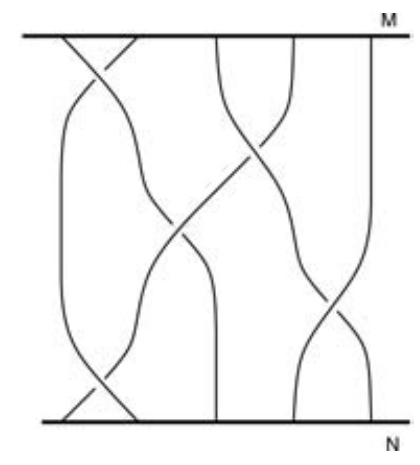

FIGURE 1. A 4-fibre braid diagram, the word representing this braid is $b_{1} b_{3} b_{2}^{-1} b_{4}^{-1} b_{1}$.

\subsection{Entanglement variable}

The inter-fibre topological structure will affect the material's response to stress. Although the inclusion of this macroscopic variable is heuristic there is a mathematical theory that can be applied to quantify the entanglement of braids. The topology of braids can be defined as a group [2]. One way to visualise the elements of an $n$-fibre braid group is to consider a braid diagram (see Figure 1). These diagrams are composed of two parallel, non-intersecting, lines denoted $\mathrm{M}$ and $\mathrm{N}$, in between which lie the fibre trajectories. The binary operation $x y$ acting on elements of the group, $x$ and $y$, can be thought of as simply attaching the fibre end-points of $y$ at $\mathrm{M}$ to those of $x$ at $\mathrm{N}$. The cross-over points between fibres in the braid diagram can be arranged so that one can define a set of $n-1$ braid generators $\left\{b_{1}, \ldots, b_{n-1}\right\}$ where for $b_{i}$ none of the fibres cross-over except for $i$ and $i+1$ strands, $i$ crosses over $i+1$. A braid can then be represented by a product of generators, which are called words, and these form elements of an $n$-fibre braid group. Note that by constraining the position of the fibre end-points of a three-dimensional braid, one may construct appropriate homotopic deformations so that a projection onto a plane corresponds to a braid diagram.

The following relationships

$$
b_{i} b_{i}^{-1}=1, \quad b_{i} b_{i+1} b_{i}=b_{i+1} b_{i} b_{i+1} \quad \text { and } \quad b_{i} b_{j}=b_{j} b_{i} \forall|i-j|>2
$$

namely, the inverse, the Artin Relationship and far commutivity, can be used to form equivalence classes and these correspond to topological equivalence. If one considered an element of a braid group with a large number of generators, possibly appearing to be highly tangled, it could be in the same equivalence class as a braid with fewer generators or even the identity braid.

The shortest word in each equivalence class gives a good indication of the degree to which fibres are entangled. The entanglement function $\kappa$ we use is proportional to the length of the shortest word within its respective equivalence class per unit volume of the continuum. We thus have a theoretical basis for our concept of "entanglement" although in practice it would require a considerable amount of effort to estimate the entanglement of a particular collection of fibres. However we can still postulate an evolution equation for the macroscopic quantity $\kappa$ which will allow us to distinguish between tufts of fibres in different states of entanglement. 


\subsection{Conservation laws}

Using the summation convention we write the conservation of mass and linear momentum,

$$
\begin{aligned}
\frac{\partial \rho}{\partial t}+\frac{\partial}{\partial x_{k}}\left(\rho u_{k}\right) & =0, \\
F_{i}+\frac{\partial \sigma_{i k}}{\partial x_{k}} & =0,
\end{aligned}
$$

where $u_{i}$ are the components of velocity, $F_{i}$ are body forces and $\sigma_{i j}$ are the bulk internal stresses due to the fibre-fibre interactions. Equation (3.2) is based on the assumption of negligible inertia due to the dominant inter-fibre frictional forces. Typical body forces $F_{i}$ due to the hooks, electro-statics, airflow and gravity could be included.

\subsubsection{Bulk stress}

The two factors that give rise to internal forces and bulk structural rigidity are individual contact points between fibres and the orientation of the fibre network. We write the bulk stress as

$$
\sigma_{\alpha \beta}=\sigma_{\alpha \beta}^{I}+\sigma_{\alpha \beta}^{N},
$$

where $\sigma^{I}$ represents the isotropic response to stress when the fibres are randomly aligned and $\sigma^{N}$ incorporates the aligned phase and hence the non-isotropic components due to the directionality of the fibres. When all the fibres are aligned, $\phi=1$, we do not expect the isotropic component of the stress $\sigma^{I}$ to contribute to the bulk stress. Conversely, when the material is randomly orientated, $\phi=0$, the directional dependent component of the stress $\sigma^{N}$ should be negligible.

The stress tensor will also depend on the degree of entanglement and initially we shall assume that the relationship is linear. Equation (3.3) should also depend on a variable that indicates the number of contact points between fibres per unit volume. Komori \& Makishima [15] have shown that in a randomly orientated collection of fibres the number of contact points between fibres is proportional to the square of density.

For small strains we expect the bulk stresses for entangled fibres to behave elastically to some degree. Whereas large strains will produce predominantly non-recoverable changes and thus a visco-elastic model would seem appropriate. However we eschew this degree of complication and present a viscous model which is applicable when the deformations are significant. This is satisfactory when considering a model for the carding machine as its objective is to dramatically alter intrinsic inter-fibre structure. As a starting point we assume that the stresses are linearly proportional to rates-of-strain and will take the form:

$$
\begin{aligned}
& \frac{\sigma_{\alpha \beta}^{I}}{\rho^{2} \kappa(1-\phi)}=\mu_{1} e_{\alpha \beta}+\mu_{2} e_{k k} \delta_{\alpha \beta}, \\
& \frac{\sigma_{\alpha \beta}^{N}}{\rho^{2} \kappa \phi}=\lambda_{1} e_{\alpha \beta}+\lambda_{2} e_{k k} \delta_{\alpha \beta}+\lambda_{3}\left(a_{\alpha} a_{i} e_{\beta i}+a_{\beta} a_{i} e_{i \alpha}\right) \\
& +\lambda_{4}\left(a_{\alpha} a_{\beta} e_{k k}+\delta_{\alpha \beta} a_{k} a_{l} e_{k l}\right)+\lambda_{5} a_{\alpha} a_{\beta} a_{k} a_{l} e_{k l}
\end{aligned}
$$


where $\mu_{i}$ and $\lambda_{i}$ are constant isotropic and anisotropic viscous coefficients respectively and $e_{\alpha \beta}=\frac{1}{2}\left(\frac{\partial u_{\alpha}}{\partial x_{\beta}}+\frac{\partial u_{\beta}}{\partial x_{\alpha}}\right)$ is the rate-of-strain tensor. We have assumed that the nematic and isotropic components of the stress depend linearly on the order parameter $\phi$ and the entanglement $\kappa$. Equation (3.5) represents a material that is transversely isotropic. The invariance of the stress tensor under isotropic rotations [11, 20, 25] leads to the particular form of the right-hand-side of (3.4) and (3.5), and thus the bulk stress depends on only 7 parameters $\lambda_{i}, \mu_{i}$.

\subsection{Kinematic condition}

In addition to conservation of mass and momentum, there is a kinematic condition to be imposed on the director field. If we consider an element of the fibres in direction $a_{i}$ with length $s$, and then impose the velocity field $u_{i}$, the director field is governed by the following equation:

$$
\frac{\partial a_{i}}{\partial t}+u_{k} \frac{\partial a_{i}}{\partial x_{k}}+\frac{\dot{s}}{s} a_{i}=a_{k} \frac{\partial u_{i}}{\partial x_{k}}
$$

where $\dot{s} / s$ is the rate-of-extension per unit length of the material. To better understand the relationship between the material element length $s$ and magnitude of stresses in the average direction of the fibres $a_{i}$, we can deduce from (3.6) that

$$
\frac{\dot{s}}{S}=a_{k} a_{l} \frac{\partial u_{k}}{\partial x_{l}},
$$

as $a_{i}$ is a unit vector. Condition (3.7) could be interpreted as a model for extensible particles but textile fibres are generally inextensible and thus the rate-of-change of our material elements $\dot{s} / s$ represents fibres slipping over one another.

\subsection{Evolution of alignment}

To encapsulate the behaviour of the alignment field we consider the result of straining some entangled fibres. There are two, not necessarily distinct, phases of interaction: the first involves the orientation of fibres in the direction of the applied force, where the inter-fibre topology remains predominantly unchanged, and in the second phase fibres slip over one another destroying the underlying inter-fibre structures. In fact the first phase would result in a change for the order parameter and the second phase a change in the entanglement. We conjecture the evolution of the order parameter to be proportional to the rate-of-extension of the material element $\dot{s} / \mathrm{s}$. Constraining the order parameter to be between zero and unity, we suggest the following simple form:

$$
\frac{\partial \phi}{\partial t}+u_{k} \frac{\partial \phi}{\partial x_{k}}=\beta(1-\phi) \frac{\dot{s}}{s}
$$

where $\beta>0$ is a parameter which we assume to be constant. We could have used other decay rates of $\phi$ but the linear form in (3.8) compares well with experimental results. Note that for this model we only consider the case where the fibres are in tension $\dot{s} / s>0$. We can eliminate $s$ by using (3.7) and re-write (3.8) as

$$
\frac{\partial \phi}{\partial t}+u_{k} \frac{\partial \phi}{\partial x_{k}}=\beta(1-\phi) a_{k} a_{l} \frac{\partial u_{k}}{\partial x_{l}} .
$$


The evolution of the order parameter is thus proportional to the magnitude of the stresses in the average fibre direction.

\subsection{Evolution of entanglement}

By assuming a simple relationship which ensures that the entanglement concentration remains positive and by considering the second phase of the simple straining experiment described above, we write:

$$
\frac{\partial \kappa}{\partial t}+u_{k} \frac{\partial \kappa}{\partial x_{k}}=-\alpha \kappa a_{k} a_{l} \frac{\partial u_{k}}{\partial x_{l}},
$$

where $\alpha>0$ is a constant parameter. We exclude the possibility of increases in entanglement and the possibility of nep formation which would occur if $\kappa \rightarrow \infty$. Note that the elongation of tufts in the carding machine will not always reduce the entanglement as some braids will need more than just simple local elongations in the average fibre direction $\boldsymbol{a}$ in order to become less or completely disentangled. Therefore this model is only valid for extensional entanglement-reducing flows. The disentangling process is not reversible, so to include increases in entanglement would be more complicated.

We now have nine field equations (3.1), (3.2), (3.6), (3.9), (3.10) and close the system with the stress tensor defined in (3.3)-(3.5). Now it remains to test the model and we consider two experiments: elongation and shearing.

\section{Parallel elongational flow}

The simplest problem which can be devised to test the model is to consider a tuft or group of entangled fibres, place parallel clamps on opposing sides, $x=0, l(0)$, then keeping one clamp fixed we elongate the material by moving the other clamp at uniform velocity $U$, see Figure 2. We expect the initial tuft, or population of tufts, to be randomly orientated and in terms of the order parameter this means that $\phi(t=0)=\phi_{0} \approx 0$. We measure the force as one clamp moves away from the other at uniform speed and use this to compare experiment with the theoretical prediction varying initial tuft lengths $l(0)$ and elongations speeds $U$.

\subsection{The governing equations}

The problem can be simplified by constraining the motion to be in one direction, so that $v, w=0$ and $\boldsymbol{a}=\boldsymbol{i}$, and the variables depend on space $x$ and time $t$ only. The governing equations, (3.1), (3.9) and (3.10), then simplify dramatically to give

$$
\begin{aligned}
\frac{\partial \rho}{\partial t}+\frac{\partial}{\partial x}(\rho u) & =0 \\
\frac{\partial \phi}{\partial t}+u \frac{\partial \phi}{\partial x} & =\beta(1-\phi) \frac{\partial u}{\partial x} \\
\frac{\partial \kappa}{\partial t}+u \frac{\partial \kappa}{\partial x} & =-\alpha \kappa \frac{\partial u}{\partial x}
\end{aligned}
$$




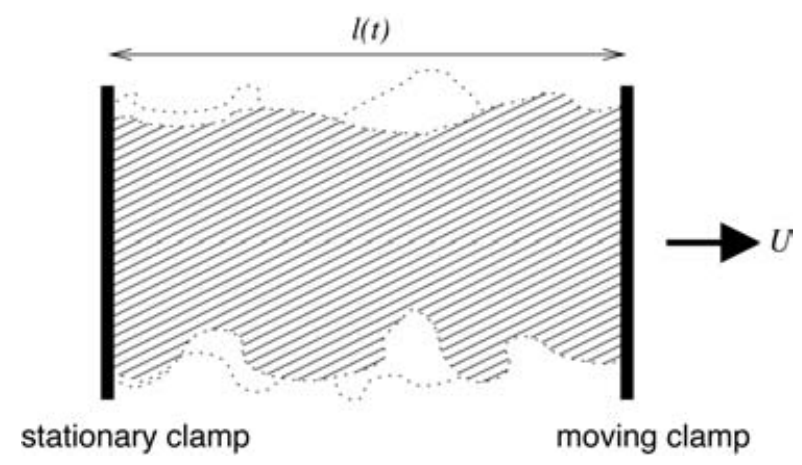

Figure 2. A diagram of unidirectional elongation.

and the equation for the divergence of the stress tensor (3.2), and the bulk stress equations (3.3)-(3.5), give

$$
\frac{\partial}{\partial x}\left(\kappa \rho^{2} \frac{\partial u}{\partial x}[A(1-\phi)+\phi B]\right)=0,
$$

where $A=\mu_{1}+\mu_{2}, B=\lambda_{1}+\lambda_{2}+2\left(\lambda_{3}+\lambda_{4}\right)+\lambda_{5}$ and $\sigma_{12}=0$.

\subsection{Boundary conditions}

At the two solid boundaries we impose no-slip velocity conditions where the boundary at $x=l(t)$ moves at a constant prescribed speed $U$, and the other at $x=0$ is fixed, so

$$
u=0 \text { at } x=0 \quad \text { and } \quad u=U \text { at } x=l(t)=l(0)+U t .
$$

Initial conditions are required for density, entanglement and order:

$$
\rho(x, 0)=\rho_{0}(x), \quad \kappa(x, 0)=\kappa_{0}(x), \quad \phi(x, 0)=\phi_{0}(x) .
$$

Note that this solution automatically satisfies the zero stress condition on the edges as $\sigma_{12}=0$ throughout.

\subsection{The solution For a uniformly dense tuft}

The solution of the differential equations (4.1)-(4.4) with conditions (4.5)-(4.6) in general will have to be computed numerically. However, if we assume that the tuft is uniform initially the equations can be solved analytically, giving the solution

$$
\begin{aligned}
\rho & =\frac{\rho_{0} l(0)}{l(0)+U t}, \quad \phi=1-\left(1-\phi_{0}\right)\left(\frac{l(0)}{l(0)+U t}\right)^{\beta}, \quad \kappa=\kappa_{0}\left(\frac{l(0)}{l(0)+U t}\right)^{\alpha}, \\
u & =\frac{U x}{l(0)+U t} \\
\text { and } F & =\frac{B \kappa_{0} \rho_{0}^{2} U}{l(0)}\left(\frac{l(0)}{l(0)+U t}\right)^{3+\alpha}\left(1-\left(1-\phi_{0}\right)\left(1-\frac{A}{B}\right)\left(\frac{l(0)}{l(0)+U t}\right)^{\beta}\right),
\end{aligned}
$$



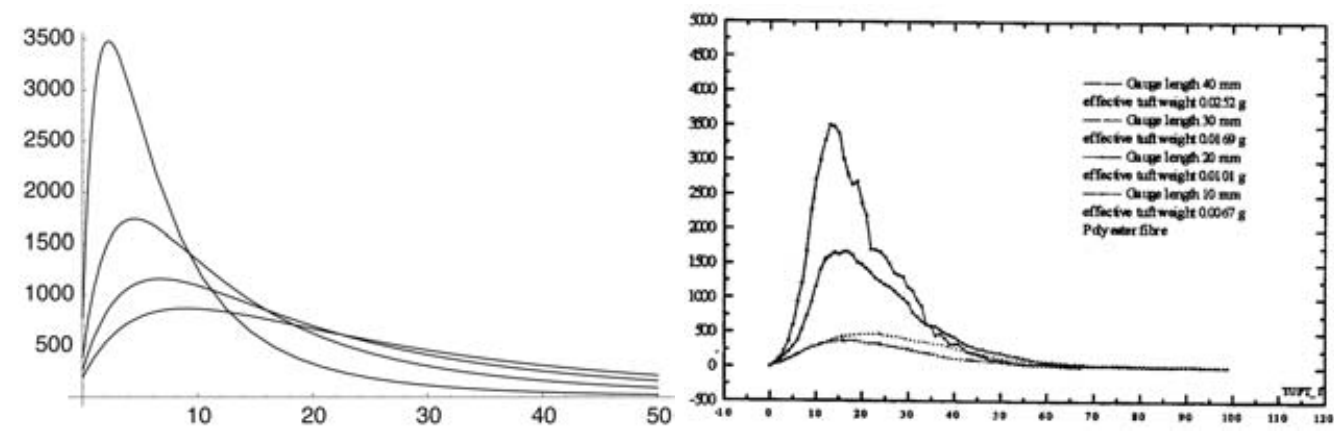

(a) Model with $l(0)=4,3,2,1 \times 10^{-2} \mathrm{~m}, U=$

(b) Experiments for polyester with $l(0)=$ $\frac{5}{6} \times 10^{-3} \mathrm{~m} / \mathrm{s}$.

\section{$4,3,2,1 \times 10^{-2} \mathrm{~m}, U=\frac{5}{6} \times 10^{-3} \mathrm{~m} / \mathrm{s}$.}
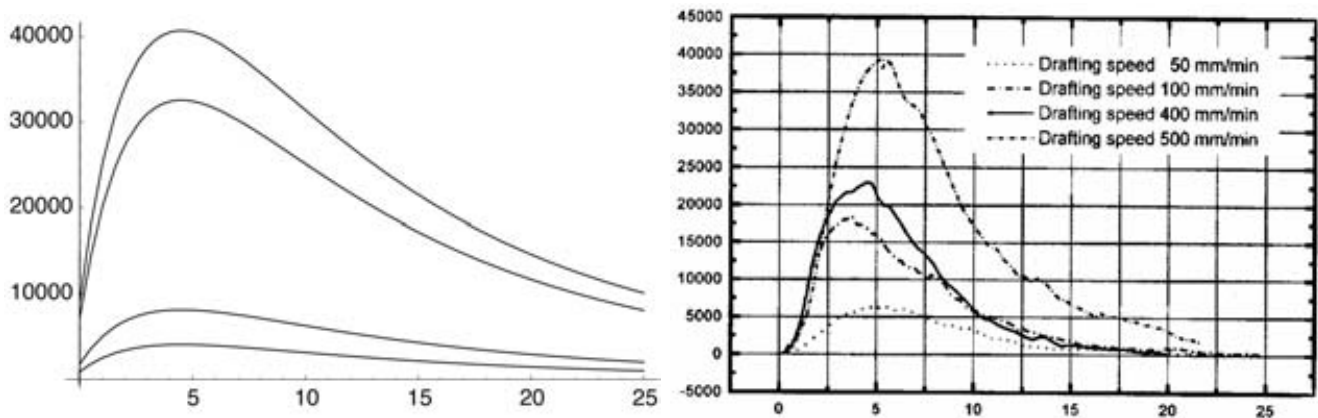

(c) Model with $U=\frac{5}{6}, \frac{10}{6}, \frac{40}{6}, \frac{50}{6} \times 10^{-3} \mathrm{~m} / \mathrm{s}$, $l(0)=2 \times 10^{-2} \mathrm{~m}$.

(d) Experiments for cotton with $U=$ $\frac{5}{6}, \frac{10}{6}, \frac{40}{6}, \frac{50}{6} \times 10^{-3} \mathrm{~m} / \mathrm{s}, l(0)=2 \times 10^{-2} \mathrm{~m}$.

Figure 3. Results of the resistive force measurements $\left(\mathrm{g} \mathrm{mm} \min ^{-2}\right.$ or $\left.\frac{10^{-8}}{36} \mathrm{~N}\right)$ plotted against elongation distance $\left(\mathrm{mm}\right.$ or $10^{-3} \mathrm{~m}$ ): (a) and (b) compares mathematical simulation with experiment for varying initial tuft lengths $l(0)$, (c) and (d) is a comparison for varying velocity $U$. The plots within each figure correlate to the given values below the respective plots in ascending order and for (a) and (c) we plot (4.7) with $\kappa_{0}=\alpha=\beta=1, \phi_{0}=\frac{A}{B}=10^{-2}$.

where $F$ is the force exerted at either end of the tuft. The density from equation (4.7) tends monotonically to zero as time tends to infinity. The order and entanglement both depend on one parameter, and their respective initial conditions; order tends to 1 and entanglement tends to 0 as $t \rightarrow \infty$. Of course in practice the tuft will have split into two pieces at some finite time.

\subsection{Comparison with experiment}

All experiments referred to in this paper were conducted by Dr Mahmoudi at the School of Textile Industries, University of Leeds. Two different fibres were used, polyester and cotton, and although they are geometrically similar, a micron in diameter and around 30 or $40 \mathrm{~mm}$ in length, one is man-made and has a smooth surface while the other is natural 
and has a hairy surface. The initial tuft sizes were varied and the experimental results are displayed in figure 3 (b) along with the mathematical prediction of the resistive force (4.7) in plot (a) for given values of $l(0)$ and $U$; assuming that the tufts are initially disordered, $\phi_{0} \ll 1$. Note that for all experimental force plots the tuft weights have been included to eliminate the differences in cross-sectional areas. The simulations are in good agreement with the experiment, but are less accurate at the beginning of the motion because we have ignored elastic effects.

In a second experiment, the velocity $U$ was varied, see figure 3 plots (c) and (d). It is quite likely that the results recorded include some noise, produced by the difficulty in obtaining equivalent tufts for each test, and in terms of the model for the same material, the initial order, entanglement and director may differ. All these parameters would also significantly change the mathematical simulation. Regardless of the possibly spurious comparative data the qualitative shape of the curves are similar in the variable velocity simulations.

The predicted theoretical force produced at the moving boundary as a consequence of the uniform elongation is given in equation (4.7). The maximum value for $F$ occurs at

$$
\frac{U}{l(0)} t_{\max }=\left(\frac{(1-A / B)\left(1-\phi_{0}\right)(3+\alpha+\beta)}{3+\alpha}\right)^{\frac{1}{\beta}}-1 .
$$

One condition for a maximum to exist when $t_{\max } \geqslant 0$ is $A<B$, and this means that the aligned viscosities should dominate over the isotropic viscosities in terms of their respective contributions to the bulk stress. Moreover, we require

$$
(1-A / B)\left(1-\phi_{0}\right)>\frac{3+\alpha}{3+\alpha+\beta},
$$

and as the disparity in the inequality (4.8) increases, $t_{\max }$ moves away from the origin, hence it seemed appropriate to choose $A / B=10^{-2}$ in the model simulations in figure 3 . For a material where $\alpha, \beta, A$ and $B$ are given then the initial order of the material is the only parameter that influences the position of the maximum, whereas the initial entanglement affects the magnitude of the resistive force linearly since the height of the maximum is

$$
F_{\max }=\frac{B \kappa_{0} \rho_{0}^{2} U}{l(0)}\left(\frac{3+\alpha}{(1-A / B)\left(1-\phi_{0}\right)(3+\alpha+\beta)}\right)^{\frac{3+\alpha}{\beta}}\left(\frac{\beta}{3+\alpha+\beta}\right) .
$$

The qualitative comparison between experiment and mathematical simulation are good. We have simply chosen $\alpha=\beta=\kappa_{0}=1$ and $B$ to match the greatest force magnitudes in corresponding mathematical and experimental results, however one could use more detailed experimental studies to estimate the parameters $\alpha, \beta, A, B$ and $\kappa_{0}$ for a chosen material.

\section{Parallel shear flow}

We continue testing the mathematical model by considering a shearing problem (see Figure 4). The material is now sheared by two parallel solid boundaries. In the experiment 


\section{moving $\longrightarrow U$}

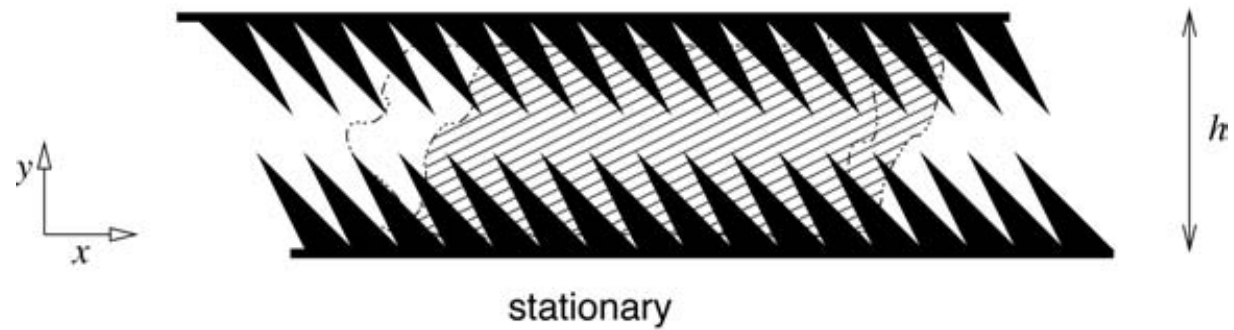

FIGURE 4. A diagram of the shearing problem.

two hooked carding surfaces were used to produce a shear stress across the tuft, one moving the other stationary. The force was measured as the moving surface travelled at uniform velocity. One method of modelling the "shear force" experiment is to consider the hooks as a body force $F_{i}$, and this is similar to ideas used by Ockendon \& Terrill [21] for a viscosity dominated Newtonian fluid moving through an array of long tethered fibres. However we shall neglect the effect of hooks in this article and address a simple channel flow.

\subsection{The governing equations}

We consider the planar problem where $w=0$ and the director is written as $\left(a_{1}, a_{2}, 0\right)=$ $(\cos \theta, \sin \theta, 0)^{T}$, we assume that the initial state is uniform in $x$ and thus the solution will depend only on variations in time and in $y$. The governing equations from (3.1), (3.6), (3.8) and (3.10) become

$$
\begin{aligned}
& \frac{\partial \rho}{\partial t}+\frac{\partial(v \rho)}{\partial y}=0 \\
& \frac{\partial \theta}{\partial t}+v \frac{\partial \theta}{\partial y}=\cos \theta \sin \theta \frac{\partial v}{\partial y}-\sin ^{2} \theta \frac{\partial u}{\partial y} \\
& \frac{\partial \phi}{\partial t}+v \frac{\partial \phi}{\partial y}=\beta(1-\phi)\left(\sin \theta \cos \theta \frac{\partial u}{\partial y}+\sin ^{2} \theta \frac{\partial v}{\partial y}\right), \\
& \frac{\partial \kappa}{\partial t}+v \frac{\partial \kappa}{\partial y}=-\alpha \kappa\left(\sin \theta \cos \theta \frac{\partial u}{\partial y}+\sin ^{2} \theta \frac{\partial v}{\partial y}\right)
\end{aligned}
$$

The conservation of momentum equations (3.2) with (3.3)-(3.5) reduce to

$$
\begin{gathered}
\frac{\partial}{\partial y}\left\{\rho ^ { 2 } \kappa \left(\frac{\partial u}{\partial y}\left[\frac{\mu_{1}}{2}(1-\phi)+\phi\left(\frac{\lambda_{1}}{2}+\frac{\lambda_{3}}{2}+\lambda_{5} \sin ^{2} \theta \cos ^{2} \theta\right)\right]\right.\right. \\
\left.\left.+\phi \frac{\partial v}{\partial y}\left[\left(\lambda_{3}+\lambda_{4}\right) \sin \theta \cos \theta+\lambda_{5} \cos \theta \sin ^{3} \theta\right]\right)\right\}=0, \\
\frac{\partial}{\partial y}\left\{\rho ^ { 2 } \kappa \left(\phi \frac{\partial u}{\partial y}\left[\left(\lambda_{3}+\lambda_{4}\right) \sin \theta \cos \theta+\lambda_{5} \sin ^{3} \theta \cos \theta\right]\right.\right. \\
\left.\left.+\frac{\partial v}{\partial y}\left[\left(\mu_{1}+\mu_{2}\right)(1-\phi)+\phi\left(\lambda_{1}+\lambda_{2}+2 \lambda_{3} \sin ^{2} \theta+2 \lambda_{4} \sin ^{2} \theta+\lambda_{5} \sin ^{4} \theta\right)\right]\right)\right\}=0 .
\end{gathered}
$$


The boundary conditions are that there is no-slip at the solid boundaries $y=0$ and $y=h$ and initial conditions are given for density, order, the director and entanglement:

$$
\begin{gathered}
u=0, v=0 \text { at } y=0, \quad u=U, v=0 \text { at } y=h, \\
\kappa(y, 0)=\kappa_{0}(y), \quad \theta(y, 0)=\theta_{0}(y), \quad \phi(y, 0)=\phi_{0}(y), \quad \rho(y, 0)=\rho_{0}(y),
\end{gathered}
$$

and these are sufficient conditions to determine the solution of equations (5.1)-(5.6). Unlike the parallel elongation problem in $\S 4$, where we had to impose a zero-stress condition transverse to the direction of elongation, we have a solid boundary that eliminates this problem, allowing $\sigma_{22} \neq 0$. The no-slip condition (5.7) is an approximation because the fibres are not clamped to the moving surfaces as they were in the parallel elongational flow problem, but they are attached to the hooks on each surface. In the experiment the fibres will begin to slip over the hooks as the density and entanglement decrease.

\subsection{The solution}

When we have constant initial conditions, we find that there is a solution for the governing equations (5.1)-(5.6), in which $v=0$ and $\kappa, \phi$ and $\theta$ are only time dependent. This allows us to integrate both equations (5.5) and (5.6) with respect to $y$ and applying the boundary conditions (5.7) gives a simple linear velocity profile,

$$
u=y \frac{U}{h} .
$$

Now, with equation (5.9), the partial differential equations in (5.2)-(5.4) become ordinary differential equations in time with the solution

$$
\begin{aligned}
& \theta=\operatorname{arccot}\left(\frac{U}{h} t+\cot \theta_{0}\right), \\
& \phi=1-\left(1-\phi_{0}\right)\left[\frac{h^{2}\left(1+\cot ^{2} \theta_{0}\right)}{h^{2}+\left(h \cot \theta_{0}+U t\right)^{2}}\right]^{\frac{\beta}{2}} \\
& \kappa=\kappa_{0}\left(\frac{1-\phi_{0}}{1-\phi}\right)^{\frac{\alpha}{\beta}}=\kappa_{0}\left[\frac{h\left(1+\cot ^{2} \theta_{0}\right)}{h^{2}+\left(h \cot \theta_{0}+U t\right)^{2}}\right]^{\frac{\alpha}{2}} .
\end{aligned}
$$

The director from (5.10), regardless of initial condition, will always tend to align itself parallel to the shearing boundaries. Order from (5.11) evolves to the aligned phase $\phi=1$ but the rate at which it evolves depends on the initial director $\theta_{0}$ and the initial order $\phi_{0}$. Similarly for the entanglement in (5.12), we see an evolution that eliminates knottedness but the rate at which this occurs depends on the initial director. The two components of force acting on the boundaries $(F, G)^{T}$ are found using the stress tensor from (5.5) and (5.6),

$$
\begin{aligned}
\frac{F(t)}{\Pi(t ; c)^{\frac{\alpha}{2}}}= & \frac{U \rho_{0}^{2} \kappa_{0} \lambda_{3}}{2 h}\left(\left[1-\left(1-\phi_{0}\right) \Pi(t ; c)^{\frac{\beta}{2}}\right]\left[\frac{1}{2}\left(1+\frac{\lambda_{1}}{\lambda_{3}}\right)+\frac{\lambda_{5}}{\lambda_{3}} \frac{h^{2}(U t+h c)^{2}}{\left(h^{2}+(U t+h c)^{2}\right)^{2}}\right]\right. \\
& \left.+\frac{\mu_{1}}{2 \lambda_{3}}\left(1-\phi_{0}\right) \Pi(t ; c)^{\frac{\beta}{2}}\right),
\end{aligned}
$$




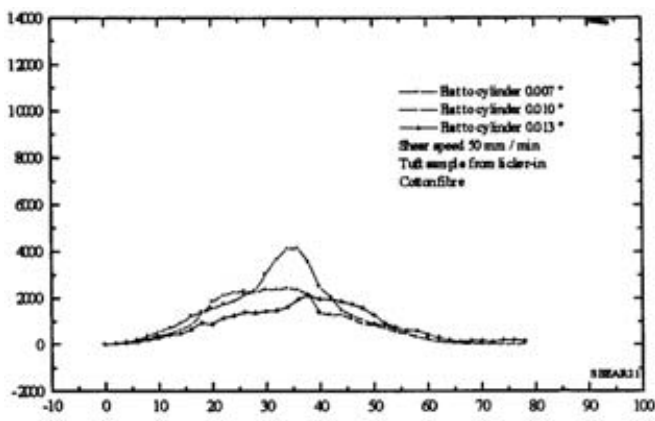

(a) Experiment with $U=\frac{5}{6} \times 10^{-3} \mathrm{~m} / \mathrm{s}$.

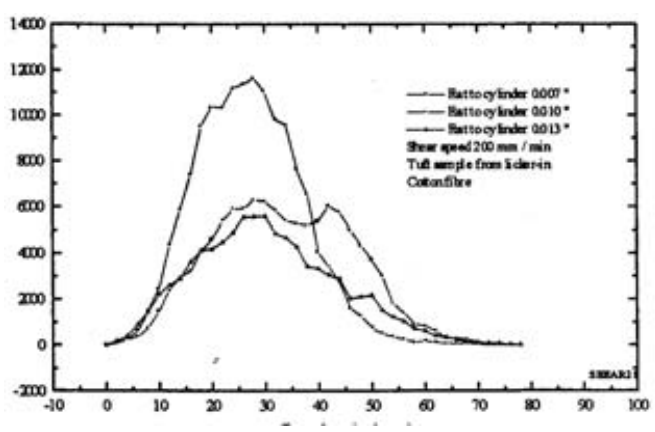

(c) Experiment with $U=\frac{20}{6} \times 10^{-3} \mathrm{~m} / \mathrm{s}$.

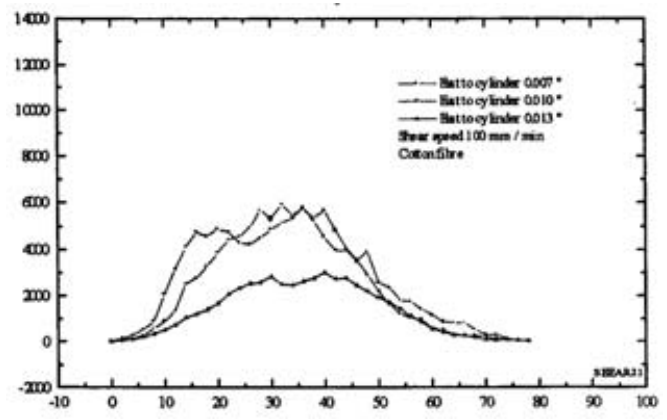

(b) Experiment with $U=\frac{10}{6} \times 10^{-3} \mathrm{~m} / \mathrm{s}$.

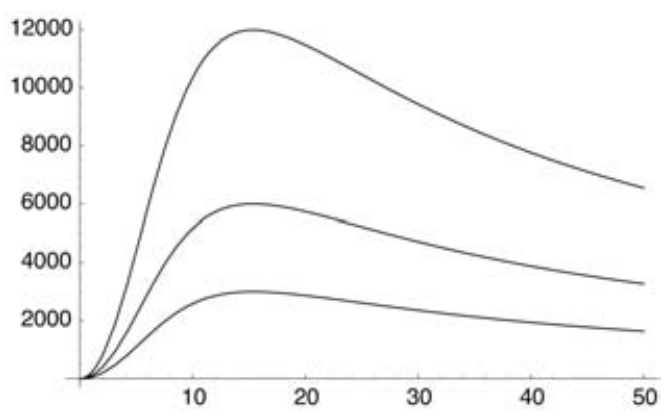

(d) Model with $U=\frac{5}{6}, \frac{10}{6}, \frac{20}{6} \times 10^{-3} \mathrm{~m} / \mathrm{s}$ in ascending order.

FIGURE 5. Results of the resistive force measurements $\left(\mathrm{g} \mathrm{mm} \min ^{-2}\right.$ or $\left.\frac{10^{-8}}{36} \mathrm{~N}\right)$ plotted against the distance sheared $\left(\mathrm{mm}\right.$ or $\left.10^{-3} \mathrm{~m}\right):(\mathrm{a})$, (b) and (c) are experiments conducted at different velocities and (d) contains theoretical plots of the force (5.13) for the three corresponding velocities with the following parameters: $h=10^{-2} \mathrm{~m}, \alpha=\beta=\frac{\lambda_{1}}{\lambda_{3}}=\frac{\lambda_{5}}{\lambda_{1}}=\kappa_{0}=1, \frac{\mu_{1}}{\lambda_{3}}=\phi_{0}=0, \theta_{0}=\frac{\pi}{2}$. Each experimental graph includes three hook-to-hook distances of $1.8,2.5,3.3 \times 10^{-4} \mathrm{~m}\left(h \sim 10^{-2} \mathrm{~m}\right)$ relating to the respective plots in descending order.

$$
\begin{aligned}
\frac{G(t)}{\Pi(t ; c)^{\frac{\alpha}{2}}}= & \frac{U \rho_{0}^{2} \kappa_{0} \lambda_{3}}{2 h}\left[1-\left(1-\phi_{0}\right) \Pi(t ; c)^{\frac{\beta}{2}}\right] \\
& \times\left[\left(1+\frac{\lambda_{4}}{\lambda_{3}}\right) \frac{h(U t+h c)}{h^{2}+(U t+h c)^{2}}+\frac{\lambda_{5}}{\lambda_{3}} \frac{h^{3}(U t+h c)}{\left[h^{2}+(U t+h c)^{2}\right]^{2}}\right]
\end{aligned}
$$

where $c=\cot \theta_{0}$ and $\Pi(t ; c)=h^{2}\left(1+c^{2}\right) /\left(h^{2}+(U t+h c)^{2}\right)$. Now it remains to compare the results with experimental data.

\subsection{Comparison with experiments}

As in $\S 4$, the experiments were conducted on cotton and polyester tufts, and so we use the result that the anisotropic viscosities dominate their isotropic counterparts $\frac{\mu_{1}}{\lambda_{3}}=0$ 
and employ similar assumptions that $\phi_{0}=0, \alpha=\beta=1$ and choose a $\lambda_{3}$ so that the magnitude of the greatest forces are approximately equivalent. The experimental measurements illustrated in Figures 5 (a), (b) and (c) show results for different shearing speeds $U$, and each figure includes three plots where the distance between hook tips have varied by increments of $7.6 \times 10^{-5} \mathrm{~m}\left(h \sim 10^{-2} \mathrm{~cm}\right)$. In Figure 5 (d) we have plotted three forces (5.13) for velocities $U=\frac{5}{6}, \frac{10}{6}, \frac{20}{6} \times 10^{-3} \mathrm{~m} / \mathrm{s}$. For clarity we have chosen not to include additional plots where the hook distances and ultimately the channel width $h$ varies by $\mathcal{O}\left(10^{-5}\right) \mathrm{m}$ as these result in relatively small changes in the predicted force.

The comparisons juxtaposed in Figure 5 are in good qualitative agreement. In particular, when comparing (a), (b) and (c) with (d) we see that the maxima approximately depend linearly on $U$. Additionally equation (5.13) predicts force to be inversely proportional to channel width $h$, and this can be seen to some extent in each experimental plot with fixed shear speed where the higher curves correspond to the smaller gap widths. However the difficulty of getting precise results from these experiments mean that we can only ascertain general trends.

A qualitative difference between the shearing experiments in Figure 5 and elongation experiments in Figure 3 is a more gradual increase to the maxima in the shearing experiment. This difference is also predicted by the mathematical model and is due to the director (5.10) not being initially aligned with the parallel shearing boundaries. The qualitative initial shape of the force plots are captured well by the model even though we have ignored elastic effects.

The main discrepancy when comparing the mathematical prediction and experiment in Figure 5 is that the experimental graphs show a greater decrease in the measured force after their respective maximums are reached. We can account for this as the mathematical model is based on an infinite channel of fibrous material and the experiments are conducted with tufts, which are just finite clusters of fibres. Furthermore, we have imposed a noslip condition on each boundary that will be violated once the density or entanglement decreases significantly.

\section{Conclusions}

In this paper, we have proposed a new continuum model for a medium composed of entangled fibres. Simplifications resulted from the assumptions that deformations in inter-fibre topology and structure were non-recoverable, friction between fibres dominated hydrodynamic forces and the fibres were always under tension. The resulting field equations (3.1)-(3.6), (3.9) and (3.10) govern an anisotropic viscous medium that was characterised by density, velocity, direction, order and entanglement. We have introduced three constitutive laws for the bulk stress, alignment and entanglement, and also suggested how braid groups can, in theory, be used to quantify entanglement density. The model's predictions were generally in good agreement with shearing and elongation experiments where velocities and lengthscales were varied.

\section{Acknowledgements}

We wish to express our gratitude to the EPSRC and Crosrol Ltd. who funded the interdisciplinary project, grant number GR/L62153, under which this work was completed. 
We are grateful to Prof. Carl Lawrence, Dr. Mahmoudi, Dr. Iype, Dr. Abbas and Mr. Greenwood of the Department of Textile Industries, University of Leeds for introducing us to the carding machine and many useful discussions. In particular we would like to thank Dr. Mahmoudi who conducted all of the experiments that are referred to in this paper. We would also like to thank the referee whose careful reading of the initial manuscript has led to the correction of a number of mistakes as well as the clarification of several issues.

\section{References}

[1] Abhiraman, A. S. \& George, W. (1973) A new aspect of the stochastic nature of carding. Textile Research J. 43(8), 452-467.

[2] Artin, E. (1965) Theory of braids. In: Lang, S. and Tate, J. T., editors, The Collected Papers of Emil Artin, pp. 446-471. Addison-Wesley.

[3] Batchelor, G. K. (1971) The stress generated in a non-dilute suspension of elongated particles by pure straining motion. J. Fluid Mech. 46(4), 813-829.

[4] Buckley, C. P. (1980) Review of the mechanical properties of fibres. In: Hearle, J. W. S., Thwaites, J. J. and Amirbayat, J., editors, NATO Advanced Study Institute on Mechanics of Flexible Fibre Assemblies (1979), NATO advanced study institute series: Series E, Applied sciences, pp. 35-49.

[5] Cherkassky, A. (1994) A two-dimensional mathematical model of the carding process. Textile Res. J. 64(3), 169-175.

[6] Cherkassky, A. (1995) Analysis of the smoothing effect of the card cylinder using simulation. Textile Res. J. 65(12), 723-730.

[7] De Gennes, P. G. \& Prost, J. (1993) The Physics of Liquid Crystals. Oxford University Press.

[8] Dehghani, A., Lawrence, C. A., Mahmoudi, M., Greenwood, B. \& C. I. (2000) An assessment of changes in the state of fibre mass during the early stages of the carding process. J. Textile Inst. 91(3), 359-373.

[9] DoI, M. \& Edwards, S. F. (1986) The Theory of Polymer Dynamics. Oxford University Press.

[10] ERICKSEN, J. L. (1991) Liquid Crystals with Variable Degree of Orientation. Archive of Rational Mechanics and Analysis, 113, 92-120.

[11] Green, A. E. \& Zerna, W. (1968) Theoretical Elasticity, 2nd ed. Oxford University Press.

[12] Gutierrez, H. M., Rust, J. P. \& Seyam, A. F. (1995) Modeling and simulation for control carding. Textile Res. J. 65(11), 638-643.

[13] Hinch, E. J. \& Leal, L. G. (1975) Constitutive equations in suspension mechanics. Part 1. General formulation. J. Fluid Mech. 71(3), 481-495.

[14] Hinch, E. J. \& Leal, L. G. (1976) Constitutive equations in suspension mechanics. Part 2. Approximate forms for a suspension of rigid particles affected by Brownian rotations. $J$. Fluid Mech., 76(1), 187-208.

[15] Komori, T. \& Makishima, K. (1977) Estimation of fibre orientation and length in fiber assemblies. Textile Res. J. 47, 309-314.

[16] Kong, L. X. \& Platfoot, R. A. (1997) Computational two-phase air/fibre flow within transfer channels of rotor spinning machines. Textile Res. J. 67(4), 269-278.

[17] Lawrence, C. A., Dehghani, A., Mahmoudi, M., Greenwood, B. \& Iype, C. (2000) Fibre dynamics in the revolving-flats card, Part 1, A critical review. AUTEX Res. J. 1(2), 64-77.

[18] LeE, M. E. M. (2001) Mathematical Models of the Carding Process. PhD thesis, University of Oxford.

[19] LesLie, F. M. (1968) Some constitutive equations for liquid crystals. Arch. Rat. Mech. \& Anal. 28, 265-283.

[20] Love, A. E. H. (1927) A Treatise on the Mathematical Theory of Elasticity, 4th ed. Cambridge University Press. 
[21] Ockendon, H. \& Terrill, E. L. (1993) A mathematical model for the wet-spinning process. Euro. J. Appl. Math. 4, 341-360.

[22] Roberts JR., W. W. (1996) Industrial fiber processing and machine design: mathematical modeling, computer simulation, and virtual prototyping. Textile Res. J. 66(4), 195-200.

[23] Rust, J. P. \& Gutierrez, H. M. (1994) Mathematical modeling and simulation for carding. Textile Res. J. 64(10), 573-578.

[24] Smith, A. C. \& Roberts JR., W. W. (1994) Straightening of crimped and hooked fibers in converging transport ducts: computational modeling. Textile Res. J. 64(6), 335-344.

[25] Spencer, A. J. M. (1972) Deformations of fibre-reinforced Materials. Oxford Science Research Papers. Oxford University Press.

[26] Toll, S. (1993) Note: On the tube model for fibre suspensions. J. Rheology, 37, 123-125.

[27] Toll, S. \& Månson, J.-A. E. (1994) Dynamics of a planar concentrated fiber suspension with non-hydrodynamic interaction. J. Rheology, 38(4), 985-997.

[28] Toll, S. \& Månson, J.-A. E. (1995) Elastic compression of a fiber network. J. Appl. Mech. 62, 223-226.

[29] Wibberly, L. D. \& Roberts JR., W. W. (1997) Modeling the diffusive transport of bulk fiber mass in a card. Textile Res. J. 67(4), 296-308. 